\title{
Synthesis of Copolymers Based on Thiophene, Methoxythiophene, Propylenedioxythiophene and Quinoxaline Units and their Optical and Electrochemical Properties
}

\author{
Dehao Kong ${ }^{1}$, Shengyong Liu ${ }^{1, *}$, Jinsheng Zhao ${ }^{2}$, Yan Zhang ${ }^{2, *}$ \\ ${ }^{1}$ School of Electric and Information Engineering, Guangxi University of Science and Technology, \\ Liuzhou, 545006, China \\ ${ }^{2}$ School of Chemistry and Chemical Engineering, Liaocheng University, Liaocheng, 252059, China \\ *E-mail: liusypp@163.com (S.Y. Liu); zhang_yan1219@126.com (Y.Zhang)
}

Received: 5 May 2021 / Accepted: 24 September 2021 / Published: 10 November 2021

\begin{abstract}
Three D-A-type conjugated copolymers namely PTQET-1, PTQET-2 and PTQET-3 were designed employing quinoxaline units as the acceptor, thiophene, methoxythiophene and 3,4propylenedioxythiophene (ProDOT) units as the donor. The three polymers had different feed ratio of the units and exhibited different electrochromic properties. They showed significant color changes during oxidation process with low optical band gaps as $1.51 \mathrm{eV}$ for PTQET-1, $1.42 \mathrm{eV}$ for PTQET-2, and $1.29 \mathrm{eV}$ for PTQET-3. In particular, PTQET-2 and PTQET-3 displayed rare neutral black color. In the kinetic experiments, the three polymers presented high coloring efficiency and optical contrast in the near-infrared region, and especially PTQET-2 showed short switching time less than $1 \mathrm{~s}$ both in the nearinfrared region and in the visible region. Besides, the polymers possessed favorable electrochromic stability and thermal stability. The new polymers reported in this paper provided a novel structural design for neutral black electrochromic materials, and they were worthy of attention and research.
\end{abstract}

Keywords: D-A type copolymers, thiophene derivatives, quinoxaline, electrochromism, neutral black

\section{FULL TEXT}

(C) 2021 The Authors. Published by ESG (www.electrochemsci.org). This article is an open access article distributed under the terms and conditions of the Creative Commons Attribution license (http://creativecommons.org/licenses/by/4.0/). 